\title{
Erratum to: Psammoma bodies in two types of human ovarian tumours: a mineralogical study
}

\author{
Fanlu Meng • Changqiu Wang • Yan Li • Anhuai Lu • \\ Fang Mei $\cdot$ Jianying Liu $\cdot$ Jingyun Du $\cdot$ Yan Zhang
}

Published online: 20 May 2015

(C) Springer-Verlag Wien 2015

\begin{abstract}
Psammoma body (PB) is a common form of calcification in pathological diagnosis and closely relevant to tumours. This paper focuses on the mineralogical characteristics of PBs in ovarian serous cancer and teratoma by using polarization microscope (POM), environmental scanning electron microscope (ESEM), micro-Fourier transform infrared spectroscopy (micro-FT-IR), transmission electron microscope (TEM), micro-area synchrotron radiation X-ray powder diffraction ( $\mu$-SRXRD) and fluorescence ( $\mu$-SRXRF). Both the PBs in tissues and separated from eight typical cases were investigated. POM and ESEM observation revealed the inside-out growth pattern of PBs. $\mu$-SRXRD and micro-FT-IR results demonstrated the dominant mineral phase of PBs in ovarian serous cancer and teratoma was $\mathrm{AB}$-type carbonate hydroxyapatite $\left(\mathrm{Ca}_{10}\left[\left(\mathrm{PO}_{4}\right)_{6-\mathrm{x}-\mathrm{y}}\left(\mathrm{CO}_{3}\right)_{\mathrm{x}}\left(\mathrm{HPO}_{4}\right)_{\mathrm{y}}\right]\left[(\mathrm{OH})_{2-\mathrm{u}}\left(\mathrm{CO}_{3}\right)_{\mathrm{u}}\right]\right.$ with
\end{abstract}

Editorial handling: L. Anhuai

Fanlu Meng and Changqiu Wang the first two authors contributed equally to this work.

The online version of the original article can be found at http://dx.doi.org/ 10.1007/s00710-014-0342-6. Due to several mistakes found in the original article, the whole article is re-published below in full with all the necessary corrections.

F. Meng $\cdot$ C. Wang $\cdot$ Y. Li $(\bowtie) \cdot$ A. Lu $(\bowtie) \cdot$ Y. Zhang

The Key Laboratory of Orogenic Belts and Crustal Evolution,

School of Earth and Space Sciences, Peking University, Beijing,

People's Republic of China

e-mail: liyan-pku@pku.edu.cn

e-mail: ahlu@pku.edu.cn

\section{F. Mei $\cdot$ J. Liu}

Pathology Department, School of Basic Medical Science, Health

Science Center of Peking University, Beijing, People's Republic of

China

J. Du

Pathology Department, Chaihu Health Center of Zhongxiang, Hubei,

People's Republic of China
$0 \leq \mathrm{x}, \mathrm{y}, \mathrm{u} \leq 2)$. As observed by ESEM and TEM, the layer-rich PBs in teratoma were up to $70 \mu \mathrm{m}$ and mainly consisted of $5 \mathrm{~nm}$-wide, 5-12 nm-long columnar crystals; the PBs in ovarian serous cancer with a maximum diameter of $35 \mu \mathrm{m}$ were composed of slightly longer columnar crystals and granulates with $20-100 \mathrm{~nm}$ in diameter. The selected area electron diffraction patterns showed dispersed polycrystalline diffraction rings with arching behavior of (002) diffraction, indicating the aggregated nanocrystals grew in the preferred orientation of (002) face. The EDX and $\mu$-SRXRF results together indicated the existence of $\mathrm{Na}, \mathrm{Mg}, \mathrm{Zn}$ and $\mathrm{Sr}$ in PBs. These detailed mineralogical characteristics may help uncover the nature of the pathological PBs in ovary.

\section{Introduction}

Psammoma bodies (PBs) is a type of pathological mineralization (calcification), which have been found in a great variety of neoplastic and non-neoplastic conditions. PBs are most commonly observed in papillary thyroid carcinoma (PTC), meningioma and papillary serous cystadenocarcinoma of ovary (Das 2009). In a review of worldwide literature (Fadare et al. 2004), $38 \%$ of patients with PBs have an associated malignancy or ovarian borderline tumour. Specifically, PBs are confined mainly to serous papillary adenocarcinoma (Sorbe \& Frankendal 1982), and $56 \%$ ovarian cystic teratoma contains tooth or calcification (Buy et al. 1989). The 5- and 10 -year survival rates were significantly different between the serous carcinomas with less than $5 \%$ area of PBs and those at least $5 \%$ area (Motohara et al. 2010).

Although PBs in ovarian tumours have been recognized for decades, the understanding of their formation mechanism remains poor. Until this century, PBs had been recognized as a reflection of dystrophic calcification resulting from cell necrosis (Ferenczy et al. 1977). In 2001, Kiyozuka et al. 
(2001) found the formation of PBs in ovarian cancer was closely related to BMP-2 and type-IV collagen. Silva et al. (2003) found hormone can affect the formation of PBs. Besides, nanobacteria may also get involved in the crystallization of PBs (Sedivy \& Battistutti 2003; Hudelist et al. 2004). All previous studies indicated that PBs were not merely the consequence of dystrophic calcification.

Unfortunately, few studies were conducted to recognize the PBs in tumours from the viewpoint of mineralogy. We believe mineralogical studies may help uncover the nature of PBs and the relationship between PB formation and tumour generation, and do necessary help for medical diagnosis and treatment. However, it is difficult to conduct formal mineralogical measurements on PBs due to the small quantity and tiny size. Besides, the intergrowth of organic tissues and PBs had been a tough problem until the separation method was proposed (Yang et al. 2008). Xylene and sodium hypochlorite solution was used to remove the organics. Although mineralogical studies of ovarian serous cancer have been conducted with this procedure (Wang et al. 2009), only one sample was sufficient enough for traditional XRD test. Besides, few mineralogical researches have been conducted on calcification in teratoma up till now.

The purpose of this study is to establish mineralogical study of PBs in ovarian serous cancer and teratoma, to compare the mineralogical characteristics of PBs in these diseases, and to discuss the mechanism of PB formation and development.

\section{Materials and methods}

\section{Sample collection and processing}

Six serous carcinoma of ovary and two mature cystic teratoma samples, which were collected from Peking University Third Hospital from year 2002 to 2012, were successively processed by formalin-fixation and paraffin-embedding at Peking University Health Science Center (PUHSC). The age of the patients with serous ovarian cancer ranges from 32 to 70 , while the teratoma patients were in twenties. The tissues were cut into serial sections by Leica Ultramicrotome and the thickness of the slices was either $4 \mu \mathrm{m}$ or $10 \mu \mathrm{m}$, depending on the analysis methods. For polarization microscope (POM), $4 \mu \mathrm{m}$-thick sections were cut, stained with hematoxylin-eosin (HE) and observed under Nikon LV100 POL.

The separation of PBs samples from the organic tissues was based on Yang et al. (2008) and conducted as follows. Slices of the serial sections were put into a $1.5 \mathrm{~mL}$ centrifuge tube and treated with xylene for $24 \mathrm{~h}, 12 \mathrm{~h}$ and $5 \mathrm{~h}$. Then graded alcohol of $100 \%, 95 \%, 90 \%, 85 \%, 70 \%, 50 \%$ and $30 \%$, was added to remove the xylene, $10 \mathrm{~min}$ for each grade. After that, the samples were washed with distilled water three times and digested in $5 \%$ sodium hypochlorite solution in $37{ }^{\circ} \mathrm{C}$ water bath for $30 \mathrm{~min}$, twice. Last, the remaining powders were washed by distilled water four times and dehydrated in graded alcohol of $50 \%, 65 \%, 75 \%, 85 \%, 95 \%, 100 \%$ and $100 \%$ for $15 \mathrm{~min}, 25 \mathrm{~min}, 35 \mathrm{~min}, 15 \mathrm{~min}, 25 \mathrm{~min}, 30 \mathrm{~min}$ and $40 \mathrm{~min}$, respectively. The powder samples were stored in alcohol under room temperature.

Methods for observation and analysis

\section{Environmental scanning electron microscope (ESEM)}

Ten-micrometre thick sections were collected on clean $20 \mathrm{~mm} \times 20 \mathrm{~mm}$ silicon slice and heated in thermostatic oven at $50{ }^{\circ} \mathrm{C}$ for $40 \mathrm{~min}$. Then all the tissues were treated with xylene twice for $24 \mathrm{~h}$ and $5 \mathrm{~h}$, respectively, and alcohol for $10 \mathrm{~min}$ and three times. The in situ samples were investigated by Quanta 200FEG (School of Physics, Peking University, live time $50 \mathrm{~s}$ ) and Quanta 650FEG (School of Earth and Space Sciences, Peking University, live time $10 \mathrm{~s}$ ), both of which were equipped with energy dispersive X-ray spectrum (EDX) detector. The operating conditions were set at $15 \mathrm{kV}$ and $110 \mathrm{~Pa}$, at low vacuum (0.1-1.5 Torr).

\section{Micro-fourier transform infrared spectroscopy (Micro-FT-IR)}

Due to the tiny amount of the sample, the powder sample was transferred by a needle onto a diamond window after drying, and flattened with the diamond sheet before test. Micro-FT-IR spectra were collected on NICOLET iN10 MX Micro-FT-IR spectrometer (College of Chemistry and Molecular Engineering, Peking University) with a resolution of $4 \mathrm{~cm}^{-1}$ in the 600 $4,000 \mathrm{~cm}^{-1}$ spectral range under transmission mode. Fitting procedure was conducted with Peakfit v4.12.

\section{Transmission electron microscope (TEM)}

Approximately $0.1 \mathrm{~mL}$ alcohol which contained powder samples after separation processing and ultrasonic dispersion was dripped onto a carbon-coated copper grid. The alcohol was absorbed by filter paper from the other side of the grid, thus the powders were attached to it. Then the prepared sample was loaded into the holder of TECNAIF 30 transmission electron microscope at School of Physics, PKU, which is equipped with an energy dispersive X-ray analysis (EDAX) detector, operating at $300 \mathrm{kV}$. The point resolution is $0.2 \mathrm{~nm}$ and the exposure time is $0.5 \mathrm{~s}$. The acquisition time of EDAX is approximately $2 \mathrm{~min}$. The data were processed and analyzed using Digital Micrograph version 3.6.5 (Gantan Ltd.).

\section{Micro-area synchrotron radiation X-ray analysis}

For micro-area synchrotron radiation X-ray powder diffraction ( $\mu$-SRXRD), about $0.1 \mathrm{mg}$ powder samples were mounted between two pieces of $3 \mathrm{M}$ films after alcohol 
volatilization. The tests were performed at BL15U, Shanghai Synchrotron Radiation Facility (SSRF) with wavelength of $0.6527 \AA$ and spot size of $10 \mu \mathrm{m} \times 10 \mu \mathrm{m}$. Standard sample of hydroxyapatite (HA) was also tested for comparison. All of the diffractograms were calibrated with standard data of $\mathrm{CeO}_{2}$ and converted to one-dimensional information by using the software of Fit2D V12.077. Further processing and analysis was performed by Datalab and Jade 5.0.

For micro-area synchrotron radiation X-ray fluorescence ( $\mu$-SRXRF), the 3M-mounted samples were reused. Operation conditions were set to $17 \mathrm{keV}$ and $4 \mu \mathrm{m} \times 4 \mu \mathrm{m}$ in spot size. All fluorescence signals were collected for $20 \mathrm{~s}$, and then the data were calibrated and fitted by using PyMca 4.4.0. HA was also tested as a standard.

For $\mu$-SRXRF mapping, the $20 \mu \mathrm{m}$-thick sections on the silicon slices, which have been tested by ESEM, were reused by transferring them onto Kapton films for test. The energy was set to $10 \mathrm{keV}$ for testing $\mathrm{Ca}, \mathrm{Zn}, \mathrm{Fe}$, and the fluorescence signal of each testing point was also collected for $20 \mathrm{~s}$. Data processing was done by pviewer at BL15U, SSRF.

\section{Results}

Distribution and morphology

All eight tumours contained numerous PBs with the diameter varying from several $\mu \mathrm{m}$ to $30-70 \mu \mathrm{m}$. The PBs presented a glassy appearance and constant extinction under crosspolarized light. These dark stained spherules were often encountered in the stroma including stromal histiocytes (Fig. 1a), fibrocytes (Fig. 1b) and cancer cell nest (Fig. 1c), whereas only a very few PBs occur around epithelial cells (Fig. 1d). As far as it could be judged from the nuclear structure and staining property, the cells around PBs did not appear to be degenerated. PBs were usually found in groups, especially in the stromal histiocytes where collagen fibers are abundant (Fig. 1a).
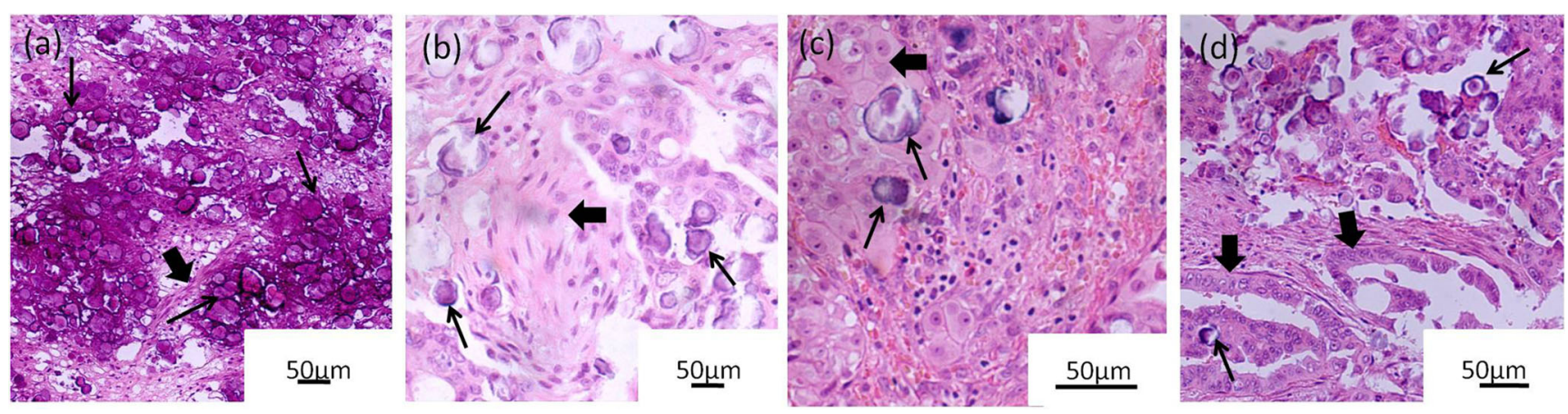

Fig. 1 Psammoma bodies in ovarian serous cancer (HE). Thin arrows point to PBs. a PBs in stromal histiocytes and collagen fibers (thick arrow). b PBs in fibrocytes (thick arrow), c PBs in cancer cells (thick arrow). d PBs in epithelial cells (thick arrow) 

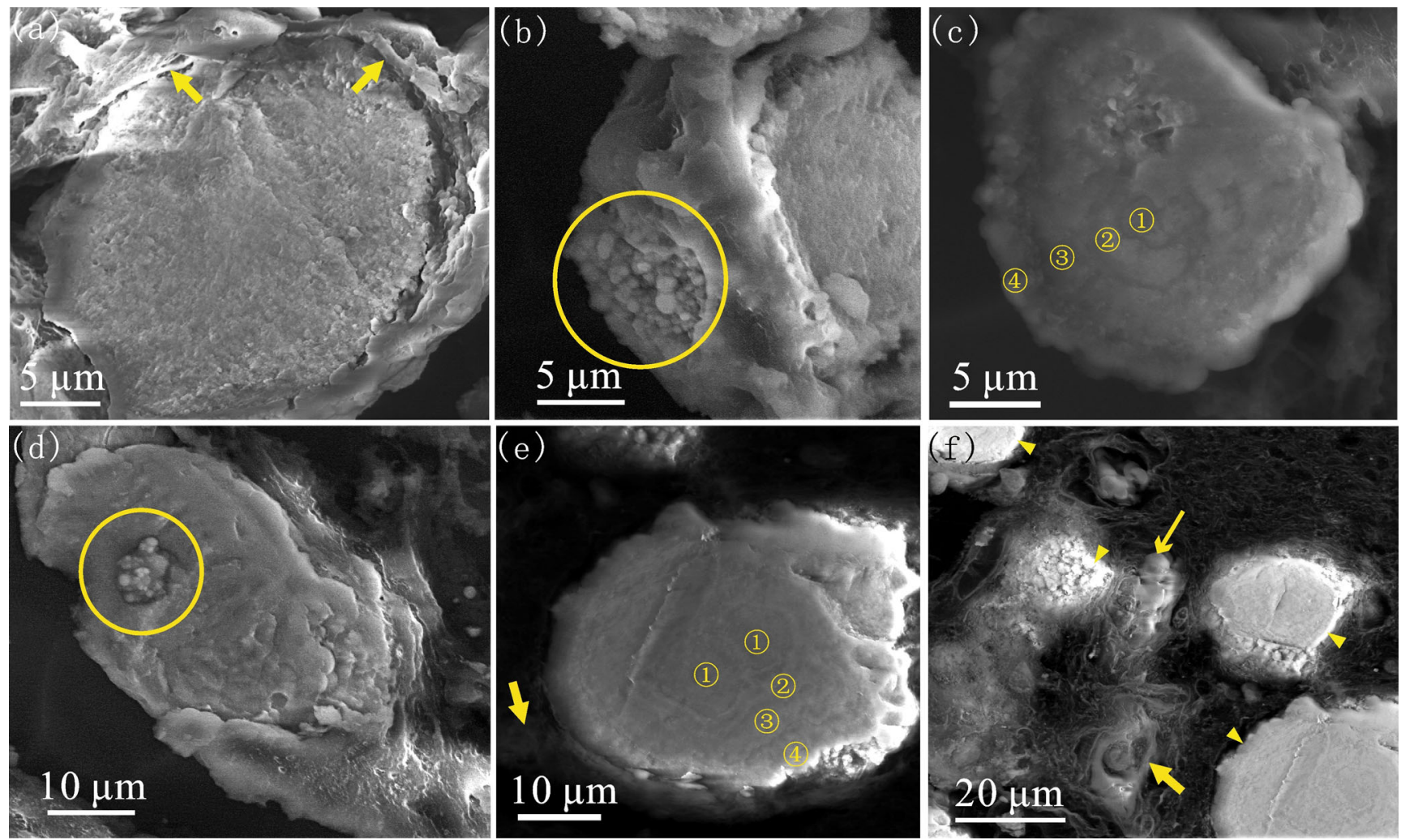

Fig. 2 ESEM images of psammoma bodies in ovarian serous cancer (ad) and teratoma (e-f). a clumpy PB covered with collagen fibers (thick arrow). $\mathbf{b}$ spherules with diameters of $40-130 \mathrm{~nm}$ on the periphery of PB (circle). c concentric layered PB with four layers. d spherules with diameters of 40-130 $\mathrm{nm}$ in the center of PB (circle). e confluent spherule

with two crystalline cores. The layered PB showed more complicated layers than that in c. Collagen fibers (thick arrow) encompass the PB. $\mathbf{f}$ blood red cells (thin arrow) and collagen fibers (thick arrow) adjacent to a cluster of PBs (arrowhead)

hydroxyapatite (replacement of $\mathrm{CO}_{3}{ }^{2-}$ for $\mathrm{OH}^{-}$) and $\mathrm{B}$ type hydroxyapatite (replacement of $\mathrm{CO}_{3}{ }^{2-}$ for $\mathrm{PO}_{4}{ }^{3-}$ ) (Mangialardo et al. 2012). The results showed the predominance of B substitution in all cases with an average B substitution/A substitution ratio of $7.1 \pm 3.3$.

TEM analysis was performed on one case of ovarian serous cancer and one case of teratoma. In ovarian serous cancer, minerals with two different morphologies were observed. One morphology was columnar crystal (Fig. 4a), which was 10 $15 \mathrm{~nm}$ in length and about $5 \mathrm{~nm}$ in width, and with a $\mathrm{Ca} / \mathrm{P}$ (At\%) ratio of 1.76. Its diffraction pattern (Fig. 4j) showed dispersed polycrystalline diffraction rings, and the lattice photograph (Fig. 4b) showed small crystalline domains, indicating the selected area is the aggregate of nanocrystals. A region of the lattice photograph was indexed after FFT, inverse FFT and calibration (Fig. 4c). Take the diffraction pattern (Fig. 4j)

into consideration, the interplanar spacing $d$ values of $0.35 \mathrm{~nm}$ and $0.28 \mathrm{~nm}$ can be assigned to the crystal faces of $(002)$ and (112) of carbonate hydroxyapatite (ICDD, 19-0272), respectively. The single crystalline diffraction behavior of (002) indicated the preferred orientation on this face (Fig. 4j). The other morphology was granulates or dumbbells with 20 $100 \mathrm{~nm}$ in diameter (Fig. 4d). The lattice photography (Fig. 4e) showed some areas of one-dimensional lattices (circles), which might be assigned to approximately $5 \mathrm{~nm}$ globules (arrow) in the center of granules. The $d$ value of the onedimensional lattices is approximately $0.35 \mathrm{~nm}$, which can be attributed to the crystal face of (002) in either hydroxyapatite $(3.44 \AA)$ or carbonate hydroxyapatite $(3.46 \AA)$. Its $\mathrm{Ca} / \mathrm{P}(\mathrm{At} \%)$ ratio was 1.87 , which was also assigned to be carbonate hydroxyapatite. In teratoma, the morphology of minerals (Fig. 4g) is similar as the columnar crystals in ovarian serous

Table $1 \mathrm{Ca} / \mathrm{P}(\mathrm{At} \%)$ ratio of each layer in concentric layered psammoma bodies

\begin{tabular}{lllllll}
\hline Tumour type & PB & Layer(1) & Layer(2) & Layer(3 & Layer(4) & Live time \\
\hline Ovarian serous cancer & PB1 & 1.32 & 1.36 & 1.46 & 1.33 & $50 \mathrm{~s}$ \\
& PB2 & 1.02 & 1.07 & 1.11 & 1.08 & $10 \mathrm{~s}$ \\
Teratoma & PB3 & 2.15 & 2.36 & 2.07 & 2.17 & $50 \mathrm{~s}$ \\
& PB4 & 1.56 & 1.89 & 1.50 & 1.62 & $50 \mathrm{~s}$ \\
\hline
\end{tabular}




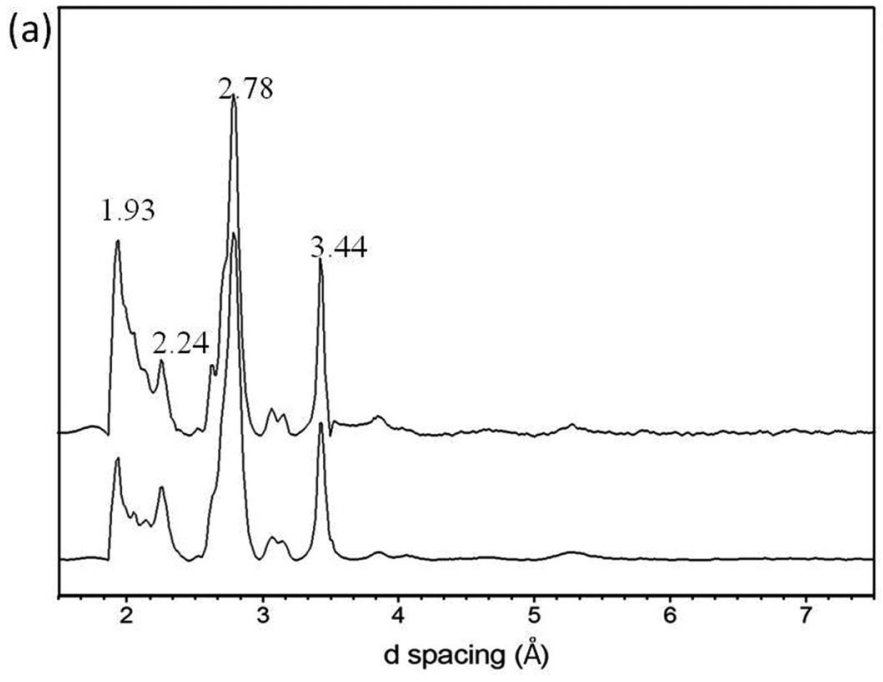

Fig. $3 \mu$-SRXRD (a) and Micro-FT-IR (b) results of PBs in ovarian serous cancer (up) and teratoma (down). a the mineral phase of PBs was identified as hydroxyapatite $\left(\mathrm{Ca}_{5}\left(\mathrm{PO}_{4}\right)_{3}(\mathrm{OH})\right.$, PDF\#09-0432) or

cancer. But the size of single crystal was slightly smaller, which was $5-12 \mathrm{~nm}$ in length and about $5 \mathrm{~nm}$ in width. Although the lattice photograph (Fig. 4h, i) showed similar interplanar spacing $d$ values with that in Fig. 4c, its diffraction pattern (Fig. 4k) showed more complicated diffraction rings and dots. The mineral phase in teratoma was identified as hydroxyapatite (ICDD, 09-0432) after calibration and indexed in Fig. 4k. It was distinguished from carbonate hydroxyapatite by visible split (circle) among (211) (2.81 $\AA$ ), (112) (2.78 $\AA$ ) and (300) $(2.72 \AA)$. Preferred orientation was also observed with the arching behavior of the (002) diffraction.

The $\mu$-SRXRF results (Fig. 5) revealed the peaks of Ca, $\mathrm{Zn}$ $\mathrm{Fe}$ and $\mathrm{Sr}$ of the PBs in ovarian serous cancer (Fig. 5a) and teratoma (Fig. 5b) as well as the expected Ti and Br introduced by $3 \mathrm{M}$ film (Fig. 5a). Compared with the curve of HA (Fig. 5b), PBs may contain certain amount of $\mathrm{Zn}, \mathrm{Fe}$ and $\mathrm{Sr}$. Further, we conducted $\mu$-SRXRF mapping on a $80 \mu \mathrm{m} \times$ $80 \mu \mathrm{m}$ region of a PB in ovarian serous cancer. The results (Fig. 6) revealed the distribution of $\mathrm{Zn}$ were generally associated with $\mathrm{Ca}$. Although Fe was shown to be in the minerals by $\mu$-SRXRF analysis, the mapping result showed a much lower counts of $\mathrm{Fe}$ in calcified region comparing with the nearby tissues. Therefore, it could not be excluded that Fe was introduced by trace amount of organic residual.

\section{Discussions}

Since few necrotic tissues were observed, our current study did not support the dystrophic mechanism of PBs. However, granules with 20-100 $\mathrm{nm}$ in diameter were observed by TEM even after separating procedures, which were previously

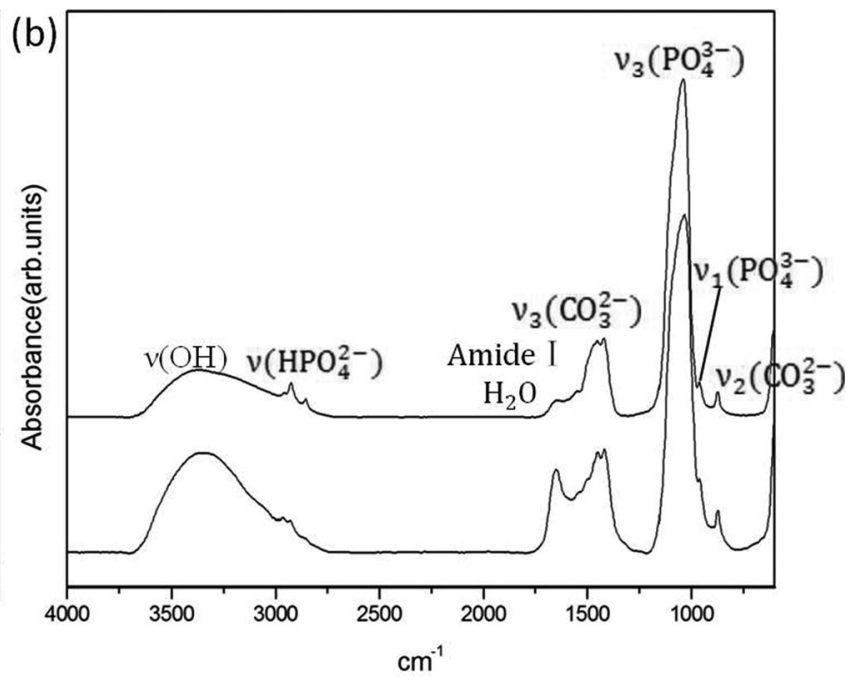

carbonate hydroxyapatite $\left(\mathrm{Ca}_{5}\left[\mathrm{PO}_{4}, \mathrm{CO}_{3}\right]_{3}(\mathrm{OH}), \mathrm{PDF} \# 19-0272\right)$. b Micro-FT-IR spectra confirmed the existence of carbonate hydroxyapatite

reported to resemble membrane bound vesicles (Ferenczy et al. 1977; Yamashima et al. 1986; Credá-Nicolás 1992). However, the nature of the granules in amorphous state is obscure. The granules can also be amorphous calcium phosphate (Jiang et al. 2012), which is a common precursor of crystalline HAPs. It is not easy to judge whether they are inorganics or undissolved organics when they intergrowth with crystalline solid. Anyway, the lattice photograph (Fig. 4e) showed crystalline globules with diameter of $5 \mathrm{~nm}$ distributed among amorphous granules, indicating a tendency of crystallization. It suggested the globules with $0.35 \mathrm{~nm} d$ spacing might be the precursors of PBs.

Since spherules in the centre and periphery of PBs were similar, in both morphology and composition, the two parts might be homologous and the layered PBs presumably resulted from coalescence and accumulation of the nano-spherules. The confluent spherules with two crystalline cores indicated the inside-out development sequence and the possibility of confluence of two PBs in later period.

The POM and ESEM of this study demonstrated the development of PBs is closely related to collagen fibers, considering their location and distribution density. Previously, many authors have claimed the importance of collagen fibers in the formation of the calcified structures in meningioma (CredáNicolás 1992; Tsuchida et al. 1996; Kubota et al. 1984). In their studies, collagen as well as elastic fibers, which laid adjacent and parallel to the crystals in the meningocytic whorls, oriented the precipitation during growth of PBs. However, these whorl structures are absent in ovarian serous cancer. And in our study, collagen fibers encompassed the large PBs optionally and irregularly, except for a case of teratoma where small calcification was surrounded by circular collagen fibers. Therefore, the morphologically controlling function of 

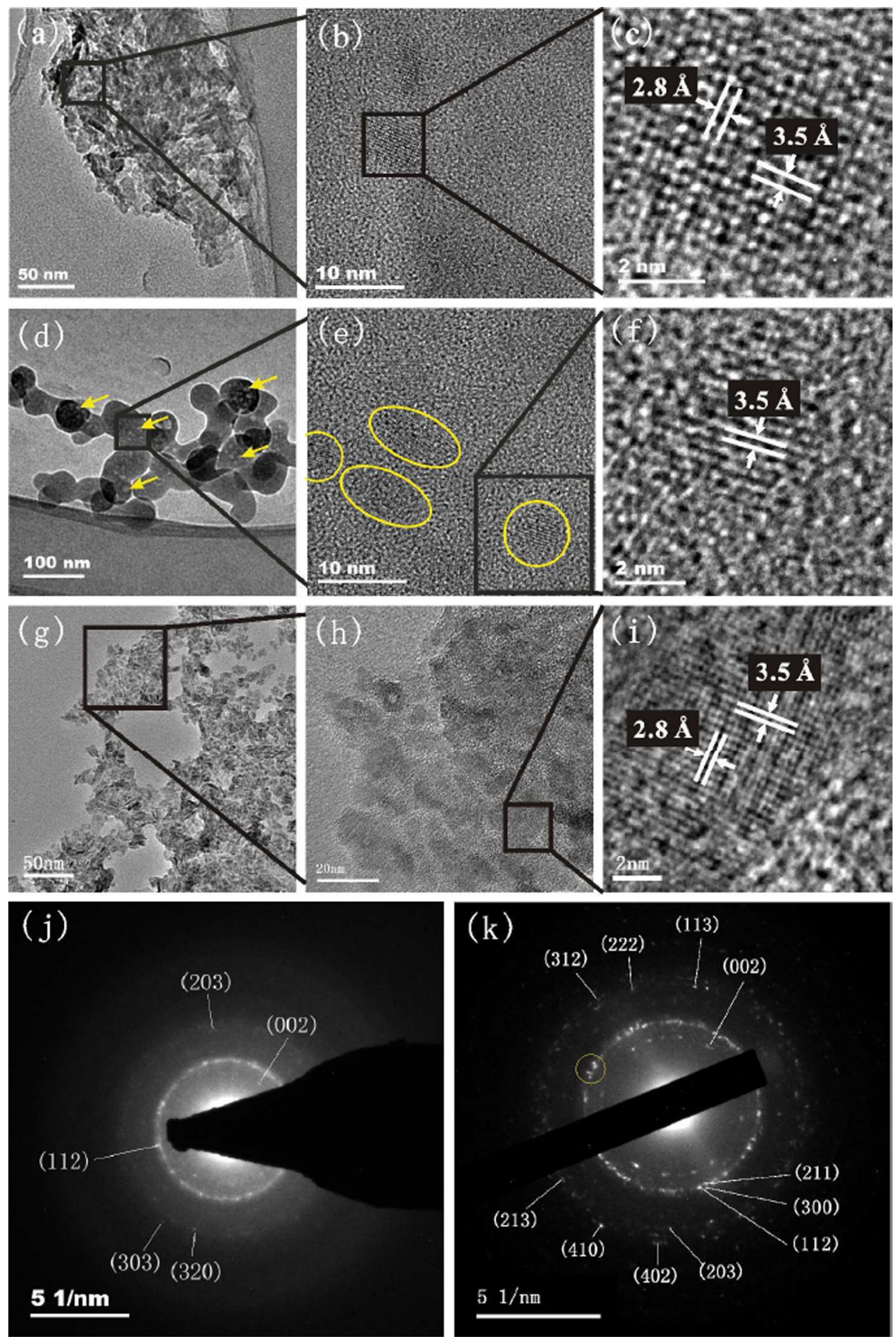

the collagen fibers might work in the initiation phase, regulating the preferred orientation on (002) face of (carbonate) hydroxyapatite (Rhee et al. 2001). In another word, the (carbonate) hydroxyapatite crystals preferred to grow along the crystallographic $c$-axis which was preferentially aligned in some certain directions. Blood cells might be a calcium source since some blood red cells presumably carrying $\mathrm{Ca}^{2+}$ could be encompassed by collagen fibers and voluminous PBs were observed in the vicinity. There could be coordination between carboxyl and carbonyl on the collagen fibers and $\mathrm{Ca}^{2+}$ and $\mathrm{Zn}^{2+}$ carried by blood or tissue fluid (Alberts et al. 1998; Rhee et al. 2000; Zhang et al. 2003). 
Fig. 4 TEM morphology (a, d, g), lattice photograph (b, c, e, f, h, i) and diffraction pattern $(\mathbf{j}, \mathbf{k})$ of the minerals in ovarian serous cancer $(\mathbf{a}-\mathbf{f}$, j) and teratoma $(\mathbf{g}-\mathbf{i}, \mathbf{k})$. a morphology of columnar crystal. b lattice photograph of selected area in a. c magnified lattice photograph of selected area in b. d morphology of granulates or dumbbells with 20$100 \mathrm{~nm}$ in diameter. Arrows point to approximately $5 \mathrm{~nm}$ globules in the center of granules. e lattice photograph of selected area in $\mathbf{d}$. Areas of onedimensional lattices were circled out., which might be assigned to $5 \mathrm{~nm}$ globules in d. f magnified lattice photograph of selected area in e. The $d$ value of the one-dimensional lattices is $0.35 \mathrm{~nm}$, which can be attributed to the crystal face of (002) in either hydroxyapatite or carbonate hydroxyapatite. $\mathrm{g}$ morphology of columnar crystals with slightly smaller size, which was $5-12 \mathrm{~nm}$ in length and about $5 \mathrm{~nm}$ in width. $\mathbf{h}$ lattice photograph of selected area in $\mathbf{g}$. i magnified lattice photograph of selected area in $\mathbf{h}$. Similar interplanar spacing $d$ values with that in $\mathbf{c}$ was observed. $\mathbf{j}$ diffraction pattern of $\mathbf{b}$ and around. $\mathbf{k}$ diffraction pattern of $\mathbf{h}$ and around

Greater odds of $c$ crystal surface exposure facilitate the absorption of protein molecule onto (carbonate) hydroxyapatite through carboxyl (and phosphate) groups (Kawasaki et al. 1985).

The mineral phase was determined comprehensively by several techniques. According to the $\mu$-SRXRD, PBs consist of either hydroxyapatite $\left(\mathrm{Ca}_{10}\left(\mathrm{PO}_{4}\right)_{6}(\mathrm{OH})_{2}\right)$ or carbonate hydroxyapatite $\left(\mathrm{Ca}_{10}\left[\mathrm{PO}_{4}, \mathrm{CO}_{3}\right]_{6}(\mathrm{OH})_{2}\right)$. Then with the help of micro-FT-IR, the presence of carbonate at both $\left[\mathrm{PO}_{4}{ }^{3-}\right]$ and $\left[\mathrm{OH}^{-}\right]$sites was verified. The existence of $\mathrm{HPO}_{4}{ }^{2-}$ was also revealed. Therefore, the dominant mineral phase of PBs in both ovarian serous cancer and teratoma can be identified as
AB-type carbonate hydroxyapatite $\left(\mathrm{Ca}_{10}\left[\left(\mathrm{PO}_{4}\right)_{6-x-}\right.\right.$ $\left.{ }_{\mathrm{y}}\left(\mathrm{CO}_{3}\right)_{\mathrm{x}}\left(\mathrm{HPO}_{4}\right)_{\mathrm{y}}\right]\left[(\mathrm{OH})_{2-\mathrm{u}}\left(\mathrm{CO}_{3}\right)_{\mathrm{u}}\right]$ with $\left.0 \leq \mathrm{x}, \mathrm{y}, \mathrm{u} \leq 2\right)$, which is commonly found in normal and pathological calcification in the human body (LeGeros 2001; Rey et al. 2007). When it comes to TEM observation, our measurement of $d$ spacing was not accurate enough to distinguish hydroxyapatite from carbonate hydroxyapatite, since they are similar to each other, especially considering the crystal face with $3.44 \AA$ spacing in hydrxyapatite and $3.46 \AA$ in carbonate hydroxyapatite. Nev-

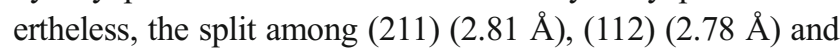
(300) (2.72 $\AA$ ) can reveal the characteristics of hydrxyapatite. In carbonate hydroxyapatite, $2.78 \AA$ should be the only peak in this range. Thus, hydroxyapatite also existed in the PBs, at least in teratoma, although carbonate hydrxyapatite was the dominant. It can be inferred that the carbonate substitution was unevenly distributed and the selected area contained less carbonate. Therefore, detailed researches of carbonate distribution will be of great help to explain the development of the minerals.

Some differences of mineralogical characteristics between PBs in ovarian cancer and those in teratoma were found in our study. The PBs in ovarian serous cancer had the maximum diameter of $35 \mu \mathrm{m}$. They consisted of columnar crystal that was $10-15 \mathrm{~nm}$ in length and $\sim 5 \mathrm{~nm}$ in width (Fig. 4a), and granulates or dumbbells with 20-100 $\mathrm{nm}$ in diameter. In teratoma, the PBs with the maximum diameter up to $70 \mu \mathrm{m}$ consist mainly of smaller columnar crystals with 5-12 nm in length and $\sim 5 \mathrm{~nm}$ in width. It is also noteworthy that the concentric layers in teratoma are more complicated than that in (a)

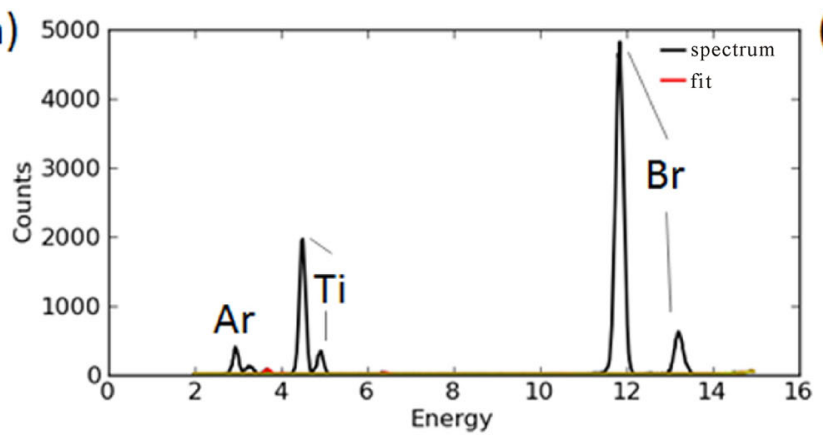

(c)

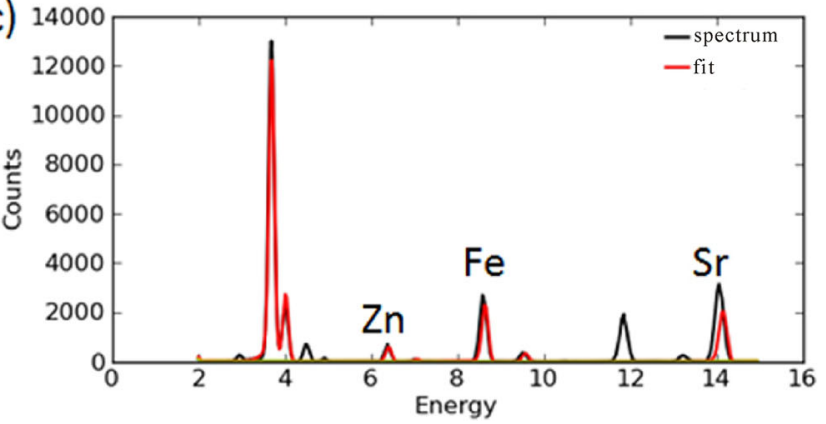

(b)

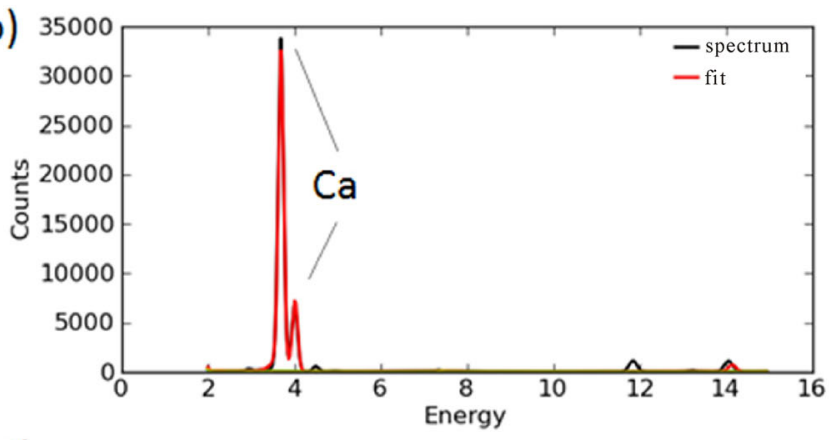

(d)

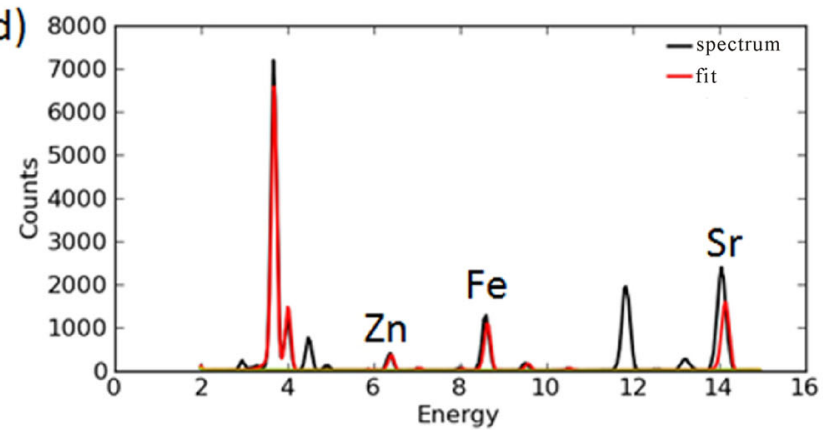

Fig. 5 Fitting curves of $\mu$ SRXRF results. a 3 M matrix, b HA, c PBs in ovarian serous cancer, d PBs in teratoma 

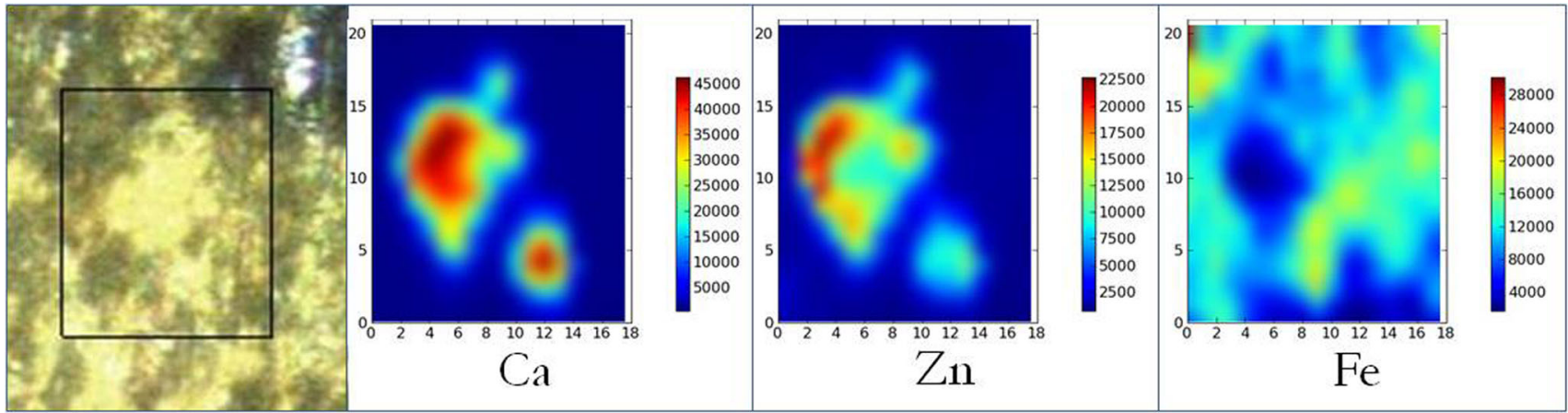

Fig. $6 \mu$ SRXRF mapping results of psammoma bodies in ovarian serous cancer. $\mathrm{Zn}^{2+}$ might substitute $\mathrm{Ca}^{2+}$ in the lattice of carbonate hydroxyapatite

ovarian serous cancer. Therefore, the crystal size and concentric structure can be important indication for physiological and pathological changes in the ovary.

$\mu$-SRXRF results revealed the existence of $\mathrm{Zn}$ and $\mathrm{Sr}$ in PBs, thus establishing more relevance between PBs and human bones which also contains additional ions. Specially, Zn plays an important role in initiation and progression of cancer (Ho 2004). Therefore, to figure out the interaction among (carbonate) hydroxyapatite, zinc and organic tissue will be helpful for oncology. According to $\mu$-SRXRF mapping results, $\mathrm{Zn}^{2+}$ might substitute $\mathrm{Ca}^{2+}$ in the lattice of carbonate hydroxyapatite. Unfortunately, the possible substitution failed to be shown by $\mu$-SRXRD results, owing to the low concentration of $\mathrm{Zn}$.

\section{Conclusions}

Mineralogical investigations were conducted on the microcalcification in PB form within human ovarian serous cancer and teratoma. The optimized separating procedure proved to be feasible to pick out the pathological calcification from the human tissues, thus overcoming the interference from the organics when investigating the mineralogical characteristics of microcalcification. Micro-area analysis, such as micro-FT-IR, TEM, $\mu$-SRXRD and $\mu$-SRXRF, was of great importance in measuring the PBs which are often in small quantity and tiny size.

The dominant mineral phase of the PBs was identified as AB-type carbonate hydroxyapatite $\left(\mathrm{Ca}_{10}\left[\left(\mathrm{PO}_{4}\right)_{6-\mathrm{x}-}\right.\right.$ y $\left.\left(\mathrm{CO}_{3}\right)_{\mathrm{x}}\left(\mathrm{HPO}_{4}\right)_{\mathrm{y}}\right]\left[(\mathrm{OH})_{2-\mathrm{u}}\left(\mathrm{CO}_{3}\right)_{\mathrm{u}}\right]$ with $\left.0 \leq \mathrm{x}, \mathrm{y}, \mathrm{u} \leq 2\right)$ with trace elements of $\mathrm{Na}, \mathrm{Mg}, \mathrm{Zn}$ and $\mathrm{Sr}$. Collagen fibers may not only be adjacent to the PBs, but also play a crucial role on the preferred orientation of (002) face and the inside-out growth pattern of PBs. The crystal size and concentric structure can be important indication for physiological and pathological changes in the ovary. The size of columnar crystals was smaller and the concentric layer was more complicated in teratoma. The reveal of extra cations and anionic groups in the microcalcification provides new clues for pathological diagnosis and detailed information of crystallization will help with either promotion or inhibition of the crystals.

Acknowledgments This work was supported by the National Basic Research Program of China (973 Program, No. 2014 CB846001) and National Natural Science Foundation of China (No. 41272048,40872196). We thank staff at BL15U, SSRF for providing beam time and offering help during the tests.

\section{References}

Alberts IL, Nadassy K, Wodak SJ (1998) Analysis of zinc binding sites in protein crystal structures [J]. Protein Sci 7(8):1700-1716

Buy JN, Ghossain MA, Moss AA, Bazot M, Doucet M, Hugol D, Truc JB, Poitout P, Ecoiffier J (1989) Cystic teratoma of the ovary: CT detection [J]. Radiology 171(3):697-701

Credá-Nicolás M (1992) Meningiomas: morphologic and ultrastructural characteristics of psammoma bodies [J]. Arch Neurobiol (Madr) 55(6):256-261

Das DK (2009) Psammoma body: a product of dystrophic calcification or of a biologically active process that aims at limiting the growth and spread of tumor? [J]. Diagn Cytopathol 37(7):534-541

Fadare O, Chacho MS, Parkash V (2004) Psammoma bodies in cervicovaginal smears: significance and practical implications for diagnostic cytopathology [J]. Adv Anat Pathol 11(5):250-261

Ferenczy A, Talens M, Zoghby M, Hussain SS (1977) Ultrastructural studies on the morphogenesis of psammoma bodies in ovarian serous neoplasia [J]. Cancer 39(6):2451-2459

Ho E (2004) Zinc deficiency, DNA damage and cancer risk [J]. J Nutr Biochem 15(10):572-578

Hudelist G, Singer CF, Kubista E, Manavi M, Mueller R, Pischinger K, Czerwenka K (2004) Presence of nanobacteria in psammoma bodies of ovarian cancer: evidence for pathogenetic role in intratumoral biomineralization [J]. Histopathology 45(6):633-637

Jiang SD, Yao QZ, Zhou GT, Fu SQ (2012) Fabrication of hydroxyapatite hierarchical hollow microspheres and potential application in water treatment [J]. J Phys Chem 116:4484-4492

Kawasaki T, Takahashi S, Ikeda K (1985) Hydroxyapatite highperformance liquid chromatography: column performance for proteins [J]. Eur J Biochem 152:361-371

Kiyozuka Y, Nakagawa H, Senzaki H, Uemura Y, Adachi S, Teramoto Y, Matsuyama T, Bessho K, Tsubura A (2001) Bone morphogenetic protein-2 and type IV collagen expression in psammoma body forming ovarian cancer [J]. Anticancer Res 21(3B):1723-1730 
Kubota T, Hirano A, Yamamoto S, Kajikawa K (1984) The fine structure of psammoma bodies in meningocytic whorls[J]. J Neuropathol Exp Neurol 43(1):37-44

LeGeros RZ (2001) Formation and transformation of calcium phosphates: relevance to vascular calcification [J]. Z Kardiol 90(3):116-124

Mangialardo S, Cottignoli V, Cavarretta E et al (2012) Pathological biominerals: Raman and infrared studies of bioapatite deposits in human heart valves [J]. Appl Spectrosc 66(10):1122-1127

Motohara T, Tashiro H, Miyahara Y, Sakaguchi I, Ohtake H, Katabuchi H (2010) Long-term oncological outcomes of ovarian serous carcinomas with psammoma bodies: a novel insight into the molecular pathogenesis of ovarian epithelial carcinoma $[\mathrm{J}]$. Cancer Sci 101(6): $1550-1556$

Rey C, Combes C, Drouet C (2007) Physico-chemical properties of nanocrystalline apatites: Implications for biominerals and biomaterials [J]. Mater Sci Eng C 27:198-205

Rhee SH, Lee JD, Tanaka J (2000) Nucleation of hydroxyapatite crystal through chemical interaction with collagen [J]. J Am Ceram Soc 83(11):2890-2892

Rhee SH, Suetsugu Y, Tanaka J (2001) Biomimetic configurational arrays of hydroxyapatite nanocrystals on bio-organics $[\mathrm{J}]$. Biomaterials 22(21):2843-2847
Sedivy R, Battistutti WB (2003) Nanobacteria promote crystallization of psammoma bodies in ovarian cancer [J]. APMIS 111(10):951-954

Silva EG, Deavers MT, Parlow AF, Gershenson DM, Malpica A (2003) Calcifications in ovary and endometrium and their neoplasms [J]. Mod Pathol 16(3):219-222

Sorbe B, Frankendal BO (1982) Prognostic importance of psammoma bodies in adenocarcinomas of the ovaries [J]. Gynecol Oncol 14(1):6-14

Tsuchida T, Matsumoto M, Shirayama Y, Kasai H, Kawamoto K (1996) Observation of psammoma bodies in cultural meningiomas: analysis of three-dimensional structure using scanning and transmission electron microscopy [J]. Ultrastruct Pathol 20(3):241-247

Wang CQ, Yang RC, Lu AH, Liu JY, Zhang B (2009) Mineralogy of psammoma bodies in human ovarian cancer [J]. Acta Petrol Mineral 28(6):617-622 (in Chinese with English abstract)

Yamashima T, Kida S, Kubota T, Yamamoto S (1986) The origin of psammoma bodies in human archnoid villi [J]. Acta Neuropathol 71(1-2):19-25

Yang RC, Wang CQ, Lu AH, Liu JY, Zhang B (2008) A study of the psammoma body mineralization in meningioma [J]. Earth Sci Front 15(6):44-53 (in Chinese with English abstract)

Zhang W, Huang ZL, Liao SS, Cui FZ (2003) Nucleation sites of calcium phosphate crystals during collagen mineralization [J]. J Am Ceram Soc 86(6):1052-1054 